\title{
Negative Energy Resonances of Bosons in a Magnetic Quadrupole Trap
}

\author{
Shahpoor Saeidian* \\ Physikalisches Institut, Universität Heidelberg, Philosophenweg 12, 69120 Heidelberg, Germany \\ Igor Lesanovsky \\ IESL - Institute of Electronic Structure and Laser, \\ FORTH - Foundation for Research and Technology, \\ P.O. Box 1527, GR-71110 Heraklion, Crete, Greece \\ Peter Schmelchent \\ Physikalisches Institut, Universität Heidelberg, Philosophenweg 12, 69120 Heidelberg, Germany and \\ Theoretische Chemie, Institut für Physikalische Chemie, \\ Universität Heidelberg, INF 229, 69120 Heidelberg, Germany
}

(Dated: May 1, 2017)

\begin{abstract}
We investigate resonances of spin 1 bosons in a three-dimensional magnetic quadrupole field. Complementary to the well-known positive energy resonances it is shown that there exist shortlived, i.e. broad, negative energy resonances. The latter are characterized by an atomic spin that is aligned antiparallel to the local magnetic field direction. In contrast to the positive energy resonances the lifetimes of the negative energy resonances decreases with increasing total magnetic quantum number. We derive a mapping of the two branches of the spectrum.
\end{abstract}

PACS numbers: 32.60.+i,33.55.Be,32.10.Dk,33.80.Ps

Magnetic traps represent a very powerful and versatile tool to study ultracold atomic (or molecular) matter. In particular they are very well-suited for miniaturization via e.g. so-called atomchips [1, 2, 3] and consequently allow a processing of matter waves at the micro or even nanoscale. The basic idea behind the control of ultracold atoms via inhomogeneous magnetic fields is very simple: The neutral atoms couple to the magnetic field via their magnetic moment. Assuming strong hyperfine interactions (compared to the magnetic interactions) the latter is due to the total angular momentum being composed of the electronic and nuclear angular momentum. Traditionally an adiabatic approximation, which reduces the vector coupling of the magnetic moment to the field to a potential term proportional to the magnitude of the field, is then employed. However, in order to understand the basic physics of (individual) atoms in magnetic traps it is likewise necessary to study the quantum dynamics of the Hamiltonian for the case of the vector coupling. A number of investigations in this direction have been performed in the past.

Firstly fundamental configurations of static fields for the trapping of ground state atoms have been explored in the late eighties and early nineties [4, 5]. Planar current geometries for microscopic magnetic traps were investigated in ref. [6]. Specifically the magnetic quadrupole field [7], the wire trap 8] and the magnetic guide as well as the Ioffe trap [9, 10, 11] have been addressed. Except for the wire trap none of these configurations allow for bound states and we observe exclusively a spectrum of resonance states. Hinds and Eberlein [9, 10] analyze the spectrum and decay rates of resonances of particles with spin $\frac{1}{2}$ and 1 in a magnetic guide by determining the phase shift of scattered waves. Potvliegé and Zehnle 11] employ the complex scaling method to obtain the widths and positions of the resonance states. More recently Lesanovsky and Schmelcher [12] studied the dynamics of neutral spin- $\frac{1}{2}$ - and spin-1-particles in a $3 \mathrm{D}$ magnetic quadrupole field, equally to ref. [7], but perform a much more extensive compilation of the properties of the resonances including the derivation of an effective scalar Schrödinger equation to describe and understand long-lived states possessing large angular momenta.

In the present work we investigate a new class of shortlived resonances possessing negative energies for the case of the 3D magnetic quadrupole field. These resonances have obviously been overlooked in previous investigations and originate from a fundamental symmetry of the underlying Hamiltonian. Their interpretation in terms of spin anti-aligned transient states in the trap is outlined.

The Hamiltonian describing the motion of a pointlike particle of mass $\mathrm{M}$ and magnetic moment $\boldsymbol{\mu}$ in a $3 \mathrm{D}$ magnetic quadrupole field $\mathbf{B}=b(x, y,-2 z)$ reads $H=\frac{\mathbf{p}^{2}}{2 M}-\boldsymbol{\mu} \cdot \mathbf{B}$. For a spin-S-particle with magnetic moment $\boldsymbol{\mu}=-\frac{g}{2} \mathbf{S}$, one obtains in atomic units

$$
H=\frac{1}{2 M}\left[\mathbf{p}^{2}+\operatorname{bg} M\left(x S_{x}+y S_{y}-2 z S_{z}\right)\right]
$$

Here $g$ is the $g$-factor of the particle. Performing the scale transformation $\bar{x}_{i}=(b g M)^{1 / 3} x_{i}$ and $\bar{p}_{i}=(b g M)^{-1 / 3} p_{i}$ and omitting the bars one obtains

$$
M(b g M)^{-2 / 3} H \rightarrow H=\frac{1}{2}\left(\mathbf{p}^{2}+x S_{x}+y S_{y}-2 z S_{z}\right)
$$


Therefore the energy level spacing scales according to $\frac{1}{M}(b g M)^{2 / 3}$. The scaled Hamiltonian $(\hbar=1)$ in spherical coordinates reads

$$
H=\frac{1}{2}\left(-\frac{\partial^{2}}{\partial r^{2}}-\frac{2}{r} \frac{\partial}{\partial r}+\frac{L^{2}}{r^{2}}+r \sin \theta K-2 r \cos \theta S_{z}\right)
$$

Here $K=\cos \varphi S_{x}+\sin \varphi S_{y}$. For a spin-1-particle, which is the case we focus on in the following except explicitly stated otherwise, we have

$$
K=\frac{1}{\sqrt{2}}\left(\begin{array}{ccc}
0 & e^{-i \varphi} & 0 \\
e^{i \varphi} & 0 & e^{-i \varphi} \\
0 & e^{i \varphi} & 0
\end{array}\right) \quad \text { and } \quad S_{z}=\left(\begin{array}{ccc}
1 & 0 & 0 \\
0 & 0 & 0 \\
0 & 0 & -1
\end{array}\right)
$$
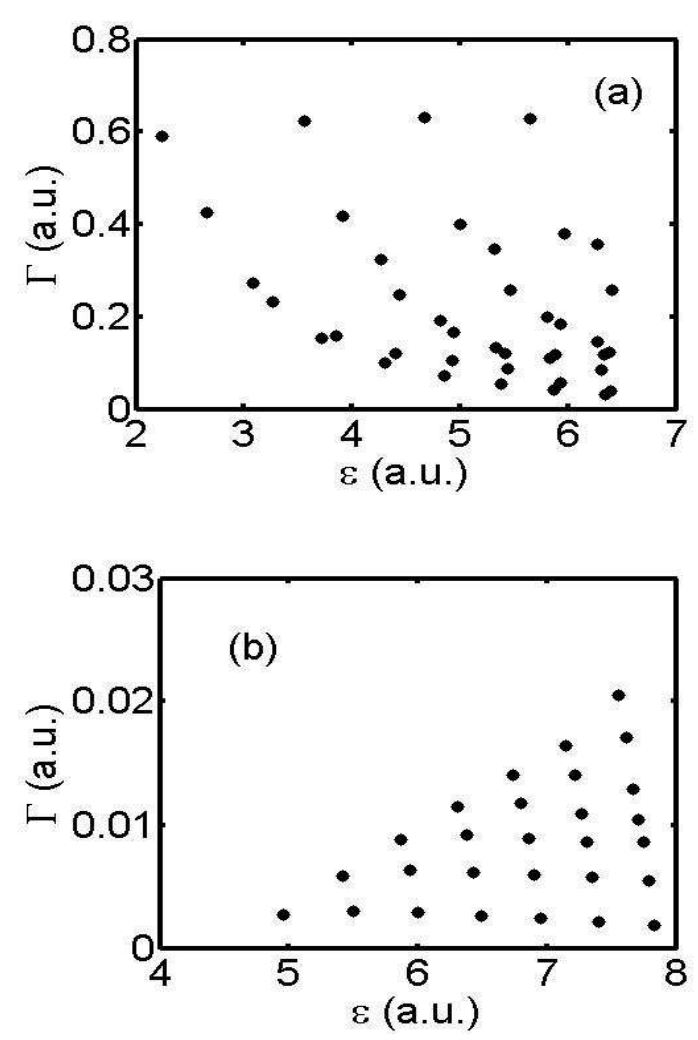

FIG. 1: Decay width and energies of the resonances possessing positive energies for (a) $m=1$ and (b) $m=10$

By analyzing the symmetry properties of the resulting Hamiltonian one obtains 15 discrete symmetries (see ref.[12]). Apart from the discrete symmetries there is a continuous symmetry generated by $J_{z}=L_{z}+S_{z}$ which is the $z$-component of the total angular momentum. This is a consequence of the rotational invariance of the system around the $z$-axis of the coordinate system. Due to $\left[J_{z}, H\right]=0$ one can find energy eigenfunctions which are simultaneously eigenfunctions of $J_{z}$. For a spin-S-particle they read in the spatial representation

$$
|m\rangle^{(s)}=\sum_{m_{s}=-s}^{s} C_{m_{s}} e^{i\left(m-m_{s}\right) \varphi}\left|m_{s}\right\rangle
$$

with $\sum_{m_{s}}\left|C_{m_{s}}\right|^{2}=1$ where $\left|m_{s}\right\rangle$ are the spin eigenfunctions with respect to $S_{z}$.

Exploiting the discrete symmetries of the system, we find a twofold degeneracy in the energy spectrum of the Hamiltonian (2) for $m \neq 0$ where $m$ is the quantum number of $J_{z}$. The corresponding eigenstates of the degenerate pair lie in $m$ - and $(-m)$-subspaces and can be identified with $|E, m\rangle$ and $|E,-m\rangle$. Of course for $m=0$ there is no degeneracy.

We are interested in the resonances of the Hamiltonian (3) which can be described by a corresponding wave function localized in space (at $t=0$ ). The time evolution is given by

$$
\psi_{R}(t)=e^{-i \frac{E}{\hbar} t} \psi_{R}(0)
$$

where $E$ is complex

$$
E=\epsilon-i \frac{\Gamma}{2}
$$

$\epsilon$ and $\Gamma$ are the energy and decay width, respectively. The imaginary part $-i \frac{\Gamma}{2}$, causes the resonances to decay with a lifetime $\tau=\Gamma^{-1}$.

In order to investigate the resonances we employ the method of complex scaling (see ref. 13] and references therein). For the computational details we refer the reader to ref. [12].

We shall see below that the spectrum consists of two well-separated parts: One set of resonances localized in the negative energy region with short lifetimes and a second set localized in the positive energy domain where the lifetimes are much larger and can, at least in principle, become arbitrarily long. The positive energy resonances have already been investigated in several works (see refs. [7, 12] and references therein) whereas, to our knowledge, no negative energy resonances have been reported up to date.

In Fig 1] we present the energies and decay widths for positive energy resonances for a spin-1-particle. For the total angular momentum $m=1$ one observes the resonances to cover the area of a right triangle in the $\epsilon-\Gamma$ plane. They are located on lines with similar negative slopes. This pattern becomes increasingly disturbed when considering resonances with higher energies and small decay widths. For higher $m$ values (see figure 1(b) for $m=10$ ) we observe a very regular pattern for their distribution in the $\epsilon-\Gamma$ plane, still with a triangular boundary, but now the resonances are located on lines possessing a positive slope (for a discussion of the origin of this regular pattern see ref. 12]). The decay width decreases exponentially with increasing value $m$ for the angular momentum. The larger the angular momentum of a state the farther away it is located from the center of the trap which is the zero of the magnetic field. In the vicinity of the center spin-flip transition from e.g. bound to unbound states take place. With increasing angular 

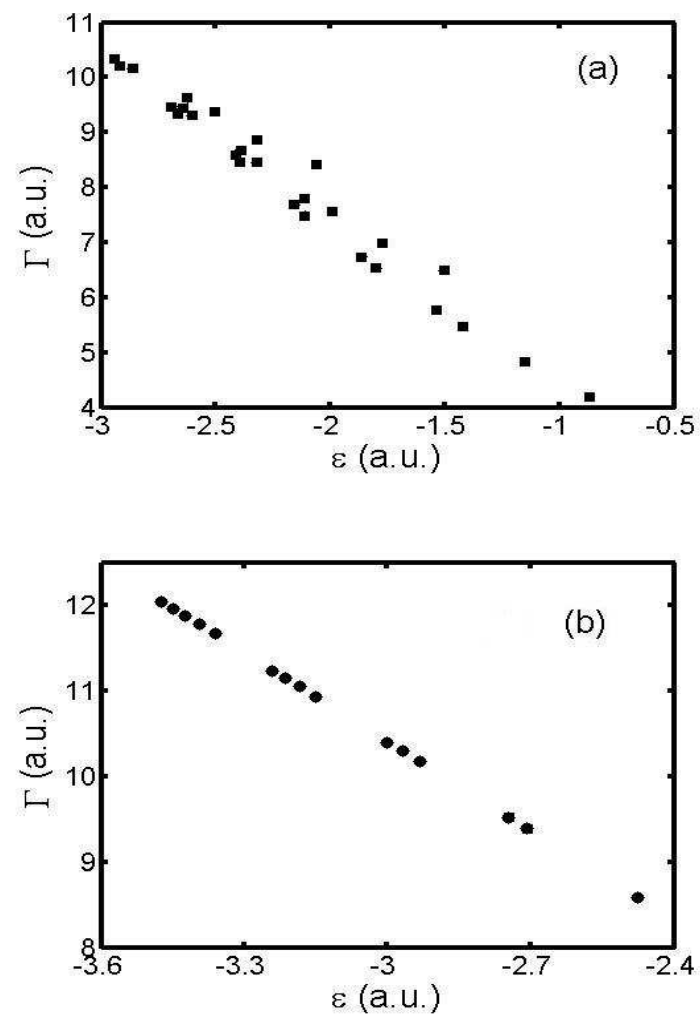

FIG. 2: Decay width and energies of the resonances possessing negative energies for (a) $m=1$ and (b) $m=10$

momentum the resonances probe less and less of this central region and become therefore increasingly stable.

Fig 2 shows the energies and decay widths for the negative energy resonances. One immediately notices that they are arranged somewhat similarly to the ones with positive energy but on lines with a negative slope. The pattern of their distribution becomes more regular for higher $m$ values. Unlike the positive energy resonances their decay width increases with increasing angular momentum. States with a larger angular momentum experience a stronger magnetic field and, as we shall show below, the magnetic moment of the atom is parallel to the local direction of the field in case of a negative energy resonance. Consequently the atom feels an increasingly repelling force with respect to the trap center if the total angular momentum increases. The latter leads to correspondingly broader resonances.

The knowledge of the resonance eigenfunctions of the complex-scaled Hamiltonian enables us to calculate corresponding expectation values of observables within the generalized inner $c$ - product being complex valued. The real part represents the average value, whereas the imaginary part can be interpreted as the uncertainty of our observable in a measurement when the system is prepared in the corresponding resonance state (for more details see [13]).
In Fig 3 we present the expectation value $\operatorname{Re}(\langle\mathbf{S}$. $\mathbf{B} /|B|\rangle)$ of the spin component which points along the local direction of the field as a function of the energy for the two cases $m=1$ and $m=10$. For the negative energy resonances this value is approximately -1 indicating that the spin is aligned opposite to the local direction of the magnetic field and the magnetic moment is in the same direction as the field (we have assumed $g>0$ ). For positive energy resonances the spin is parallel to the local field and the magnetic moment is antiparallel.

It is instructive to consider the average value of the radial coordinate $r$ as a function of the energy (see Fig 4) . The resonances are distributed in an area possessing the shape of a triangle. Again this pattern becomes increasingly regular, when considering higher angular momenta (see fig $4(b)) . R e(\langle r\rangle)$ increases with increasing absolute value of the energy which is in agreement with our above argumentation with respect to the localization of the resonances.
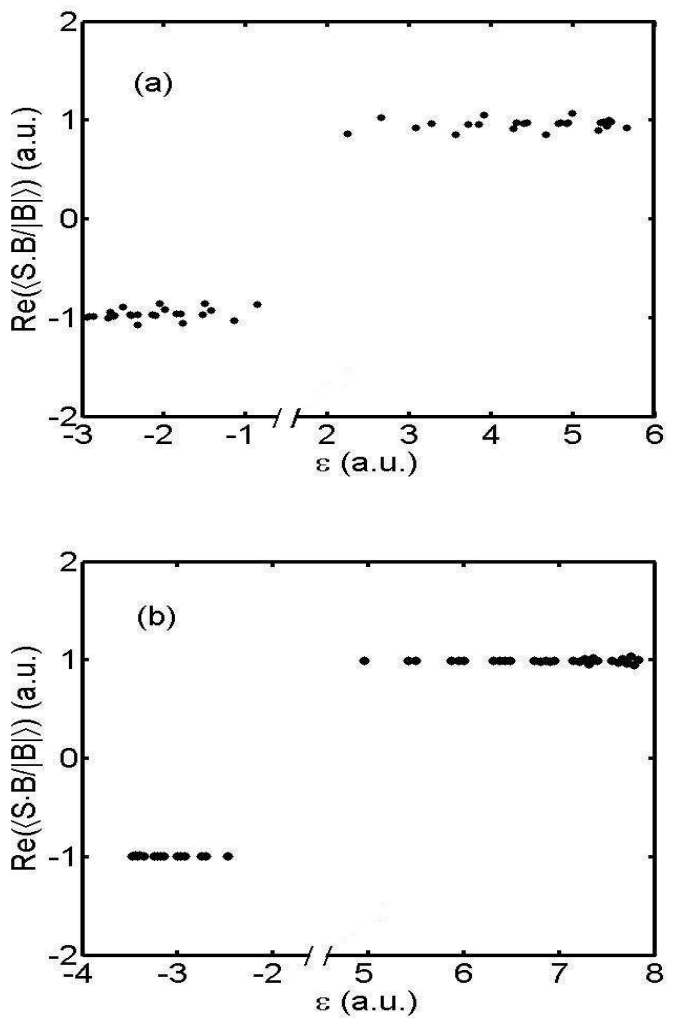

FIG. 3: Expectation values of the spin component along the local direction of the magnetic field for the positive and negative energy resonances for (a) $m=1$ and (b) $m=10$.

The results on the negative and positive energy resonances suggest an intimate relationship among the two. Indeed due to a complex symmetry, which we shall derive in the following, there exists a mapping of the two classes of resonances. The Schrödinger equation belonging to the Hamiltonian which results when applying the 
complex scaling to the Hamiltonian (3) reads

$$
\begin{gathered}
\frac{1}{2}\left(-e^{-i 2 \eta} \frac{\partial^{2}}{\partial r^{2}}-e^{-i 2 \eta} \frac{2}{r} \frac{\partial}{\partial r}+e^{-i 2 \eta} \frac{m^{2}}{r^{2}}+e^{i \eta} r \sin \theta K\right. \\
\left.-2 e^{i \eta} r \cos \theta S_{z}\right) \psi_{E, m}=E \psi_{E, m}
\end{gathered}
$$

Taking the complex conjugate and multiplying by $e^{-i \frac{2 \pi}{3}}$ one obtains

$$
\begin{gathered}
\frac{1}{2}\left(-e^{-i 2(\pi / 3-\eta)} \frac{\partial^{2}}{\partial r^{2}}-e^{-i 2(\pi / 3-\eta)} \frac{2}{r} \frac{\partial}{\partial r}\right. \\
+e^{-i 2(\pi / 3-\eta)} \frac{m^{2}}{r^{2}}+e^{i(\pi / 3-\eta)} e^{-i \pi} r \sin \theta K^{*} \\
\left.-2 e^{i(\pi / 3-\eta)} e^{-i \pi} r \cos \theta S_{z}^{*}\right) \psi_{E, m}^{*}=e^{-i \frac{2 \pi}{3}} E^{*} \psi_{E, m}^{*}
\end{gathered}
$$

By transforming

$$
\begin{gathered}
S_{i} \rightarrow \bar{S}_{i}=e^{-i \pi} S_{i}^{*}=-S_{i}^{*} \\
\eta \rightarrow \bar{\eta}=\pi / 3-\eta
\end{gathered}
$$

one can show that

$$
\begin{gathered}
\frac{1}{2}\left(-e^{-i 2 \bar{\eta}} \frac{\partial^{2}}{\partial r^{2}}-e^{-i 2 \bar{\eta}} \frac{2}{r} \frac{\partial}{\partial r}+e^{-i 2 \bar{\eta}} \frac{m^{2}}{r^{2}}+e^{i \bar{\eta}} r \sin \theta \bar{K}\right. \\
\left.-2 e^{i \bar{\eta}} r \cos \theta \bar{S}_{z}\right) \bar{\psi}_{\bar{E}, m}=\bar{E} \bar{\psi}_{\bar{E}, m}
\end{gathered}
$$

which is unitarily equivalent to eq.(7) with the transformed energies and resonance wave functions

$$
\begin{gathered}
E \rightarrow \bar{E}=e^{-i \frac{2 \pi}{3}} E^{*} \\
\psi_{E, m} \rightarrow \bar{\psi}_{\bar{E}, m}\left(e^{i \bar{\eta}} r, \theta, \varphi, m_{s}\right)=\psi_{E, m}^{*}\left(e^{i \eta} r, \theta, \varphi,-m_{s}\right)
\end{gathered}
$$

Therefore each resonance belonging to eq.(7) with eigenvalue $E$ possesses a counterpart i.e. a 'partner resonance' with the eigenvalue $\bar{E}=e^{-i 2 \pi / 3} E^{*}$. We therefore have a mirror symmetry of the underlying Hamiltonian the corresponding mirror line being placed at $-\pi / 3$. While the positive energy continuum rotates clockwise around its zero energy threshold by $2 \eta$ its image rotates anticlockwise by $2 \eta$ starting from the line defined by the polar angle $-\frac{2 \pi}{3}$. When a resonance is revealed at the angle $-2 \eta_{0}$ in the positive energy domain, its image resonance for negative energies is revealed at the polar angle position $-2 \pi / 3+2 \eta_{0}$.

Employing the transformation (9), the expectation values of $r$ and of the spin-component along the local direction of the magnetic field $\frac{\mathbf{S} \cdot \mathbf{B}}{|\mathbf{B}|}$ transforms as follows
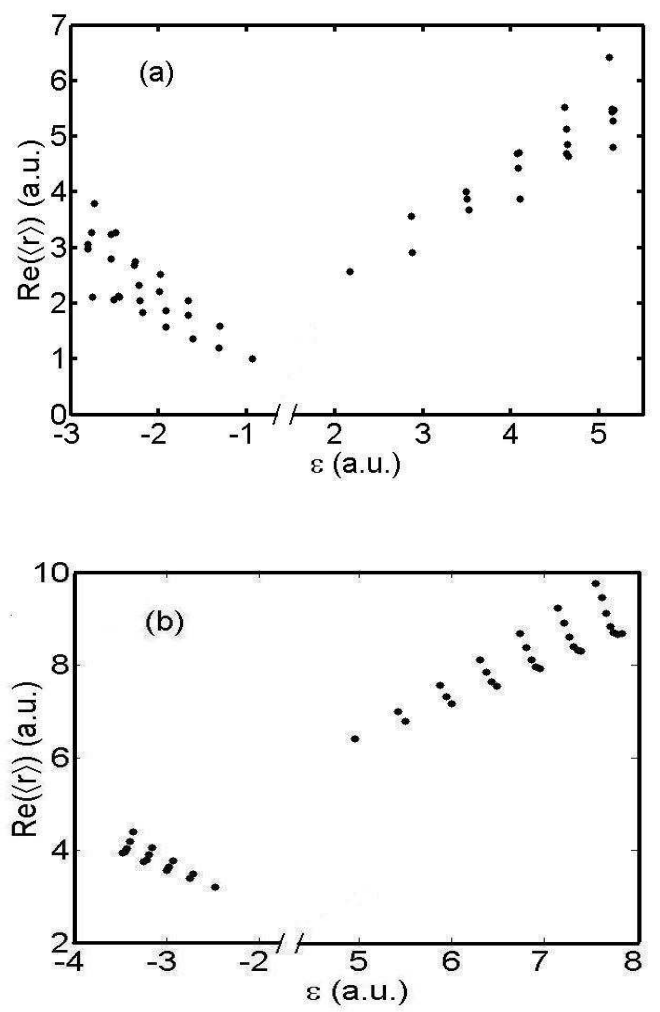

FIG. 4: Average of the radial coordinate $r$ as a function of the energy for both positive and negative energy resonances for (a) $m=0$ (b) $m=10$.

$$
\begin{gathered}
\langle r\rangle \rightarrow e^{i \pi / 3}\langle r\rangle^{*} \\
\left\langle\frac{\mathbf{S} \cdot \mathbf{B}}{|\mathbf{B}|}\right\rangle \rightarrow e^{i \pi}\left\langle\frac{\mathbf{S} \cdot \mathbf{B}}{|\mathbf{B}|}\right\rangle^{*}=-\left\langle\frac{\mathbf{S} \cdot \mathbf{B}}{|\mathbf{B}|}\right\rangle^{*}
\end{gathered}
$$

which are in good agreement with the results of our numerical calculations. Finally we remark that although our study was performed for the specific case of a spin-1particle our conclusions hold for any boson with non-zero spin and also for fermions.

Let us conclude. We observe, to our knowledge, for the first time negative energy resonances of spin-1-bosons in a 3D quadrupole magnetic trap. The overall spectrum is arranged in two disconnected parts each of which contains exclusively resonances with positive and negative energies. The latter are exclusively of short-lived character and the spin is antiparallel to the local direction of the magnetic field while for the former the spin is parallel to the field. As the total angular momentum of the boson increases, the decay width of a negative energy resonance state increases while for a positive energy resonance it decreases (assuming a typical laboratory gradient field $5 T / m$ and the species $R b-87$ the order of magnitude for the lifetimes of the negative energy resonances is mikroseconds). A property of the complex 
scaled Hamiltonian has been established which allows to map the two branches of the spectrum.

S.S. acknowledges financial support by the Ministry of Science, Research and Technology of Iran in the form of a scholarship.

* sंsaeid@physi.uni-heidelberg.de

$\dagger$ igor@iesl.forth.gr

¥ Peter.Schmelcher@pci.uni-heidelberg.de

[1] R. Folman et al, Adv.At.Mol.Phys. 48, 263 (2002)

[2] J. Reichel, Appl.Phys.B 74, 469 (2002)

[3] J. Fortagh and C. Zimmermann, Science 307, 860 (2005)

[4] W. Ketterle and D.E. Pritchard, Appl.Phys. B 54, 403 (1992)
[5] T. Bergeman, G. Erez and H.J. Metcalf, Phys.Rev. A 35 , 1535 (1987)

[6] J.D. Weinstein and K.G. Libbrecht, Phys. Rev. A 52, 4004 (1995)

[7] T.H.Bergeman et al, J.Opt.Soc.Am.B 6, 2249 (1989)

[8] K.Berg-Sorensen, M.M.Burns, J.A.Golovchenko and L.V.Hau, Phys.Rev.A 53, 1653 (1996)

[9] E.A.Hinds and C.Eberlein, Phys. Rev. A 61, 033614 (2000)

[10] E.A.Hinds and C.Eberlein, Phys. Rev. A 64, 039902(E) (2000)

[11] R. M. Potvliege and V. Zehnlé, Phys. Rev. A 63, 025601 (2001)

[12] I.Lesanovsky, P.Schmelcher, Phys. Rev. A 71, 032510 (2005)

[13] N.Moiseyev, Phys. Rep. 302, 211 (1998) 